\title{
Incidence and risk factors for febrile neutropenia in Japanese patients with non-Hodgkin B cell lymphoma receiving R-CHOP: 2-year experience in a single center (STOP FN in NHL 2)
}

\author{
Masahiro Yokoyama $^{1} \cdot$ Yoshiharu Kusano $^{1} \cdot$ Anna Nishihara ${ }^{1} \cdot$ Norihito Inoue $^{1} \cdot$ Noriko Nishimura $^{1}$ - Yuko Mishima ${ }^{1}$. \\ Yasuhito Terui $^{1} \cdot$ Tomoyuki Nukada $^{2} \cdot$ Takanobu Nomura $^{2} \cdot$ Kiyohiko Hatake $^{1,3}$
}

Received: 17 December 2018 / Accepted: 5 April 2019/Published online: 15 May 2019

(C) The Author(s) 2019

\begin{abstract}
Background Myelosuppressive chemotherapy-induced febrile neutropenia (FN) is a life-threatening condition. Patients receiving granulocyte colony-stimulating factors (G-CSF) have shorter duration of neutropenia, faster recovery from fever, and shorter duration of antibiotics use. Most strategies for FN prevention using daily G-CSF and pegfilgrastim are based on overseas studies. Data on Japanese patients were lacking; thus, we previously determined the incidence of FN in non-Hodgkin B cell lymphoma (B-NHL) patients at our center. Here, we aimed to gain additional insights into pegfilgrastim use in this population.

Methods This single-center, retrospective, observational study (STOP FN in NHL 2) enrolled patients with B-NHL who underwent a regimen comprising rituximab and CHOP therapy over a 2-year period (January 2015-June 2017). The incidence of FN in cycle 1 of chemotherapy, risk factors for FN development, and use of daily G-CSF and pegfilgrastim were evaluated. Results We evaluated 239 patients: 61 patients did not receive G-CSF and 178 received G-CSF. The incidence of FN was $10.5 \%$ (95\% confidence interval [CI] 6.9-15.1\%) in cycle 1 and $13.0 \%$ (95\% CI 9.0-17.9\%) in all cycles. The FN incidence was significantly lower $(P=0.0008)$ in patients receiving daily G-CSF and pegfilgrastim than patients not receiving G-CSF. Significant risk factors for FN were age $\geq 65$ years, albumin $<3.5 \mathrm{~g} / \mathrm{dL}$, hemoglobin $<12 \mathrm{~g} / \mathrm{dL}$, and no prophylaxis with daily G-CSF/pegfilgrastim during cycle 1 .

Conclusions The incidence of FN in cycle 1 and in all cycles and the identified risk factors were similar with those we previously reported; thus, our results validate previous findings.

Trial registration UMIN000029534.
\end{abstract}

Keywords Febrile neutropenia · Incidence $\cdot$ Japan $\cdot$ Non-Hodgkin B cell lymphoma $\cdot$ R-CHOP $\cdot$ Risk factor

Findings from this study were presented as a poster presentation at the MASCC/ISOO 2018 Annual Meeting in Vienna, Austria, held on 28-30 June 2018.

Electronic supplementary material The online version of this article (https://doi.org/10.1007/s00520-019-04802-4) contains supplementary material, which is available to authorized users.

Masahiro Yokoyama

masahiro.yokoyama@jfcr.or.jp

1 Division of Hematology Oncology, The Cancer Institute Hospital, Japanese Foundation for Cancer Research, 3-8-31 Ariake, Koto-ku, Tokyo 135-8550, Japan

2 Medical Affairs, Kyowa Hakko Kirin Co., Ltd., Tokyo, Japan

3 Department of Hematology, International University of Health and Welfare, Tokyo, Japan

\section{Background}

Myelosuppressive chemotherapy-induced febrile neutropenia (FN) is a life-threatening condition associated with increased morbidity and mortality $[1,2]$ that often results in extended hospitalization and death [3]. FN may lead to unwanted chemotherapy dose reductions or may halt treatment altogether, which can compromise treatment outcomes [2].

According to the current Japanese guidelines [4], all patients who present an FN incidence $\geq 20 \%$ should receive prophylaxis granulocyte colony-stimulating factor (G-CSF), regardless of the presence or absence of risk factors, in order to prevent and treat FN induced by chemotherapy [5]. Further, all patients with risk factors for FN and FN incidence of 10 to $<20 \%$ should receive prophylaxis with G-CSF [4]. 
A recent meta-analysis of 30 randomized trials evaluated the relative efficacy of G-CSF products as primary prophylaxis for cancer patients receiving myelosuppressive chemotherapy. The meta-analysis concluded that the risk of FN was reduced with pegfilgrastim, filgrastim, lenograstim, and lipegfilgrastim when compared with no G-CSF or placebo. Although the use of filgrastim was heterogenous across the different trials, pegfilgrastim reduced FN risk compared with filgrastim (odds ratio $=0.61 ; 95 \%$ credible interval $0.40-0.98$ ) [6]. Moreover, several trials have demonstrated that patients receiving G-CSF had a shorter duration of neutropenia, faster recovery from fever, and shorter duration of antibiotics use [7].

One cohort study identified that having non-Hodgkin lymphoma (NHL) and comorbidities such as anemia, HIV infection, and rheumatoid disease significantly increased the risk of developing FN [8]. Specifically, among patients receiving cyclophosphamide, doxorubicin, vincristine, and prednisone (CHOP) chemotherapy, characteristics including age $\geq$ 65 years, renal disease, cardiovascular disease, baseline hemoglobin $<12 \mathrm{~g} / \mathrm{dL},>80 \%$ planned CHOP average relative dose-intensity, and no G-CSF prophylaxis were significantly associated with increased risk of FN [9]. However, the data obtained only corresponded to patients who received daily G$\mathrm{CSF}$, and there is no information on risk factors of $\mathrm{FN}$ in Japanese patients receiving pegfilgrastim. Identification of characteristics that predispose patients to $\mathrm{FN}$ who require treatment with these drugs will allow an optimal prophylactic use of G-CSF, thus improving chemotherapy delivery and patient outcomes [10].

Most strategies for FN prevention using daily G-CSF and pegfilgrastim are based on data from overseas clinical studies [11]. Additionally, the frequency of FN in clinical studies may differ from that in routine clinical practice. A recent systematic review reported that a $13 \% \mathrm{FN}$ rate $(95 \%$ confidence interval [CI] 8.7-17.9\%) in a randomized clinical trial translated into a $20 \% \mathrm{FN}$ rate in an observational study [12]. Therefore, it is very important to evaluate G-CSF use and G-CSF treatment outcomes in routine clinical practice in Japan in order to achieve effective FN prevention. Recently, we conducted a retrospective, observational study in a single center in Japanese patients with non-Hodgkin B cell lymphoma (BNHL) and found that the incidence of FN was 9.1\% (42 of 462 patients) in cycle 1 and $12.3 \%$ (57 of 462 patients) throughout all cycles, with $73.7 \%(42 / 57)$ of patients developing FN during cycle 1 [13].

Pegfilgrastim has a long half-life and is administered during the early phase of a treatment cycle, usually on the day following chemotherapy, and daily G-CSF is administered mostly to patients with signs of FN [14]. A lower dose (3.6 mg) of pegfilgrastim is used in Japan [4] compared with other countries $(6 \mathrm{mg})$. However, our previous retrospective study in patients with B-NHL was conducted before pegfilgrastim became available in Japan [13], and no information was obtained on how both G-CSFs were actually administered in routine clinical practice. The present study aimed to gain additional insights into the use of pegfilgrastim, which had not been approved at the time of the previous study. Thus, we evaluated the incidence of FN in B-NHL patients who received rituximab and $\mathrm{CHOP}$ (R-CHOP regimen), identified risk factors for $\mathrm{FN}$ development by patient characteristics and FN onset, and evaluated the use of daily G-CSF and pegfilgrastim in routine clinical practice in Japan.

\section{Materials and methods}

\section{Study design and treatment}

In this single-center, retrospective, observational study (STOP FN in NHL 2), we retrospectively analyzed data from patients who underwent R-CHOP therapy over a 2-year period (between January 2015 and June 2017) at the Cancer Institute Hospital of the Japanese Foundation for Cancer Research, Tokyo, Japan.

This study was conducted in accordance with the Declaration of Helsinki and the Ethical Guidelines for Epidemiological Studies. The Ethical Review Board of our institute approved the study protocol. Based on those guidelines, informed consent from subjects was not required because the data analyzed were obtained from medical records. This trial was registered at the UMIN Clinical Trial Registry under the identifier UMIN000029534.

According to routine clinical practice in Japan, patients were hospitalized to receive cycle 1 of $\mathrm{R}-\mathrm{CHOP}$ regimen. Whether patients received daily G-CSF or pegfilgrastim, as well as the corresponding dosing, was decided by each patient's treating physician.

\section{Patients}

Patients with malignant NHL who started and completed R$\mathrm{CHOP}$ regimen during the study period, and patients who received at least three cycles of R-CHOP regimen were included in the study. Patients with HIV-related malignant lymphoma were excluded.

\section{Assessments}

The investigational items related to the patients were age, sex, performance status, body mass index, characteristics of the disease (disease name, stage, and presence or absence of bone marrow infiltration), presence or absence of complications (diabetes mellitus, hepatic, renal or cardiac diseases, uncured wounds, and others), presence or absence of previous illness (surgery, infections, and FN within 1 month before initiation of the most recent R-CHOP regimen), and blood laboratory 
parameters (albumin, total bilirubin, hemoglobin, absolute neutrophil count [ANC], and absolute leukocyte count $[A L C])$. The investigational items related to the chemotherapy cycle were number of days to the next cycle and relative dose intensity (RDI), which represented the mean RDI for cyclophosphamide and doxorubicin and was calculated as follows: [(actual dose) / (planned dose)] / [(actual duration of treatment) / (planned duration of treatment)]. The investigational items related to the development of FN were body temperature, neutrophil count, prophylactic or therapeutic intervention (oral antibiotics, G-CSF, and treatment date from the initiation of chemotherapy), and whether or not hospitalization was required.

\section{Study endpoints}

\section{Primary endpoint}

The incidence of FN in cycle 1 of chemotherapy was the primary endpoint. FN was defined as having an axillary temperature $\geq 37.5^{\circ} \mathrm{C}$ and neutropenia with an $\mathrm{ANC}$ of $<500 \mu \mathrm{L}$ or an $\mathrm{ANC}$ of $<1000 / \mu \mathrm{L}$ that is expected to decrease to $<500 /$ $\mu \mathrm{L}$ within $48 \mathrm{~h}$.

\section{Secondary endpoints}

The secondary endpoints were incidence of FN throughout all chemotherapy cycles, incidence of FN in cycle 1 of chemotherapy by patient demographic and clinical characteristics, risk factors involved in the onset of FN in cycle 1, relationship between the incidence of FN in cycle 1 and ALC, proportion of patients using antibiotics due to $\mathrm{FN}$, proportions of patients with RDI $\geq 85 \%$ and RDI $<85 \%$, and use of daily G-CSF and pegfilgrastim and their effects in routine clinical practice in Japan.

\section{Statistical analyses}

The analysis set was defined as all patients enrolled in the study. Summary statistics such as the number of patients, mean, and standard deviation were calculated for continuous variables, and discrete variables were summarized by frequency. Continuous variables were examined using a $t$ test or $u$ test depending on the data distribution. Discrete variables were examined by chi-square test. The incidence of FN throughout all chemotherapy cycles was calculated as the percentage of patients who developed FN among all patients receiving RCHOP regimen, and the corresponding 95\% CIs were calculated.

No significance level was specified as this was not a hypothesis-testing study; however, a two-sided significance level of 5\% was used for exploratory statistical analysis. We estimated the odds ratios in a logistic regression model. We then constructed a multivariate model based on the statistically relevant indicators $(P<0.10)$ found to influence the development of FN in a univariate model. These factors were then used in a stepwise variable analysis with an entry and removal probability of 0.20 to avoid overlooking factors affecting the development of FN. All statistical analyses were performed using SAS Versions 9.3 (SAS Institute, Inc., Cary, NC, USA).

\section{Simulation analysis}

The simulation analysis has been previously described in detail [13]. Briefly, 1000 bootstrap samples were built by resampling from the population analyzed for the incidence of FN in cycle 1. For each bootstrap sample, the incidence of FN in cycle 1 without prophylaxis and with G-CSF was estimated for all patients included in the bootstrap samples. We obtained 1000 estimates of the incidence of FN in cycle 1 without prophylaxis and with G-CSF from the bootstrap samples. These estimates were then used to calculate the mean, which was used as the point estimate of the incidence of FN in cycle 1 without prophylaxis and with G-CSF for all patients included in the population analyzed for the incidence of FN in cycle 1. A CI was constructed based on the 2.5th and 97.5th percentiles of the estimated incidence of FN over 1000 bootstrapped samples. All analyses were performed without applying any imputation approach to deal with the missing data.

\section{Results}

\section{Patient characteristics and treatment exposure}

A total of 239 patients were evaluated, including 61 patients who were not treated with G-CSF and 178 patients who received G-CSF. Among 178 patients, 124 patients and 54 patients were administered daily G-CSF and pegfilgrastim, respectively.

Table 1 shows the main demographic and clinical characteristics of patients. Patients had a mean age of $64.0 \pm$ 12.7 years and $46.9 \%$ were male. Most patients (95.8\%) had an Eastern Cooperative Oncology Group performance status between 0 and 1, and the most common pathological diagnosis (66.9\% of patients) was diffuse large B cell lymphoma.

The mean start date of daily G-CSF and pegfilgrastim treatment in cycle 1 was $10.18 \pm 2.67$ days and $2.59 \pm 1.39$ days, respectively, with the mean number of daily G-CSF dosing days of $2.98 \pm 1.58$ days. Online resource 1 shows the distribution of patients according to the day of G-CSF treatment start. In cycle 1, a small percentage of patients $(2.4 \%)$ treated with daily G-CSF started treatment on days 0 to 3, while most patients $(90.7 \%)$ treated with pegfilgrastim started treatment between days 0 and 3 . 
Table 1 Demographic and clinical characteristics of patients in cycle 1

$N=239$

Age (years)

$64.0 \pm 12.7$

Sex (male)

Body mass index $\left(\mathrm{kg} / \mathrm{m}^{2}\right)$

ECOG PS $\quad 0-1$

2-4

Pathological diagnosis

DLBCL

Follicular lymphoma

Transformed DLBCL

Others

$112(46.9)$

$22.6 \pm 3.4$

$229(95.8)$

$10(4.2)$

$160(66.9)$

$41(17.2)$

$13(5.4)$

$25(10.5)$

Stage

I-II

108 (45.2)

III-IV

$131(54.8)$

Bone marrow infiltration

Complications

Diabetes

$29(12.1)$

$21(8.8)$

Hepatic or renal

$13(5.4)$

Other

$127(53.1)$

Albumin (g/dL)

$3.8 \pm 0.6$

$0.6 \pm 0.3$

$12.5 \pm 2.0$

$4.2 \pm 2.0$

$1.4 \pm 1.4$

$88.7 \pm 12.5$

Relative dose intensity (\%)

Data are presented as $n(\%)$ or mean $\pm \mathrm{SD}$

ECOG PS, Eastern Cooperative Oncology Group performance status; $D L B C L$, diffuse large B cell lymphoma; $S D$, standard deviation

\section{Endpoints}

For all patients $(N=239)$, the incidence of $\mathrm{FN}$ was $10.5 \%$ (95\% CI 6.9-15.1\%) in cycle 1 (primary endpoint) and $13.0 \%$ (95\% CI 9.0-17.9\%) in all cycles of chemotherapy.

The incidences of FN in cycle 1 were compared by type of treatment (i.e., without G-CSF treatment, with daily G-CSF and with pegfilgrastim). The incidence of $\mathrm{FN}$ was significantly lower in patients treated with daily G-CSF and pegfilgrastim, compared with patients without G-CSF treatment $(7.3 \%, 3.7 \%$, and $23.0 \%$, respectively; $P=0.0008)$ (Fig. 1).

Table 2 shows the incidence of FN in cycle 1 stratified by patient characteristics. The incidence of FN was significantly higher in patients aged $\geq 65$ years vs $<$ 65 years, those with albumin $<3.5 \mathrm{~g} / \mathrm{dL}$ vs $\geq 3.5 \mathrm{~g} / \mathrm{dL}$, those with hemoglobin $<12 \mathrm{~g} / \mathrm{dL}$ vs $\geq 12 \mathrm{~g} / \mathrm{dL}$, those who used antibiotics vs those who did not used antibiotics, and those who did not use G-CSF in cycle 1 vs those who used G-CSF in cycle 1. There were no differences in the incidence of FN by sex, Eastern Cooperative Oncology Group performance status, body mass index, type of lymphoma, disease stage, presence or absence of bone marrow infiltration or complications, total bilirubin, RDI of cyclophosphamide or doxorubicin, ANC, or ALC. The proportion of patients with RDI $\geq$ $85 \%$ and RDI $<85 \%$ was $68.6 \%$ (164/239) and $31.4 \%$ (75/239), respectively. The incidences of FN in cycle 1 were compared by ALC levels (i.e., $<1.0 \times 10^{9} / \mathrm{L}, 1.0$ to $<2.0 \times 10^{9} / \mathrm{L}$, and $\geq 2.0 \times 10^{9} / \mathrm{L}$ ); there was a significantly higher incidence of FN in the group with ALC $<1.0 \times$ $10^{9} / \mathrm{L}$ compared with the groups with ALC 1.0 to < $2.0 \times 10^{9} / \mathrm{L}$ and $\geq 2.0 \times 10^{9} / \mathrm{L}(17.9 \%, 6.5 \%$, and $6.5 \%$, respectively; $P=0.0228$ ) (Fig. 2).

To identify the risk factors involved in the onset of FN in cycle 1 , we conducted a univariate analysis of patient characteristics. The statistically and clinically relevant factors identified for cycle 1 were then used for a multivariate analysis (Table 3). Age $\geq 65$ years, albumin $<3.5 \mathrm{~g} / \mathrm{dL}$, hemoglobin $<$ $12 \mathrm{~g} / \mathrm{dL}$, and lack of prophylaxis with daily G-CSF and pegfilgrastim during cycle 1 were identified as significant risk factors for FN.

\section{Simulation analysis}

As a result of the simulation analysis by multivariate analysis, four candidate risk factors for FN were identified (Table 4). Age $\geq 65$ years, albumin $<3.5 \mathrm{~g} / \mathrm{dL}$, hemoglobin $<12 \mathrm{~g} / \mathrm{dL}$, and lack of prophylaxis with daily G-CSF or pegfilgrastim were identified as significant risk factors for the development of FN in cycle 1 . These factors were then used to estimate the incidence of $\mathrm{FN}$ by multivariate logistic regression in patients without prophylactic administration of G-CSF. According to the simulation analysis, in patients who were not treated with G-CSF, the estimated incidence of FN in cycle 1 was $30.4 \%$ (95\% CI 17.2-43.1\%) (Table 4).

\section{Discussion}

Data on the efficacy of daily G-CSF vs pegfilgrastim for the prevention of FN are limited, particularly in Japanese patients. To the best of our knowledge, this is the first study to evaluate the risk factors of FN in Japanese patients receiving daily G$\mathrm{CSF}$ and pegfilgrastim in routine clinical practice. Although we had conducted a previous study that was limited to the evaluation of the effect of the prophylactic use of daily G-CSF and detection of risk factors for developing $\mathrm{FN}$ in cycle 1 in the same study center [13], the present study differs in that this study evaluated the data after pegfilgrastim became available in Japan and it includes subgroup analyses by the type of G-CSF used (daily G-CSF and pegfilgrastim) and treatment start date. Thus, we consider that the present results are a more accurate depiction of the use of G-CSF in clinical practice in Japan.

The incidence of FN in cycle 1 of chemotherapy (10.5\%) and in all cycles (13.0\%) was not different from that reported in our previous study ( $9.1 \%$ in cycle 1 and $12.3 \%$ in all cycles) 
Fig. 1 Incidence of febrile neutropenia in cycle 1 by type of treatment. $P=0.0008$ for with daily G-CSF and with pegfilgrastim vs without G-CSF (chi-squared test). G-CSF, granulocyte colony-stimulating factor

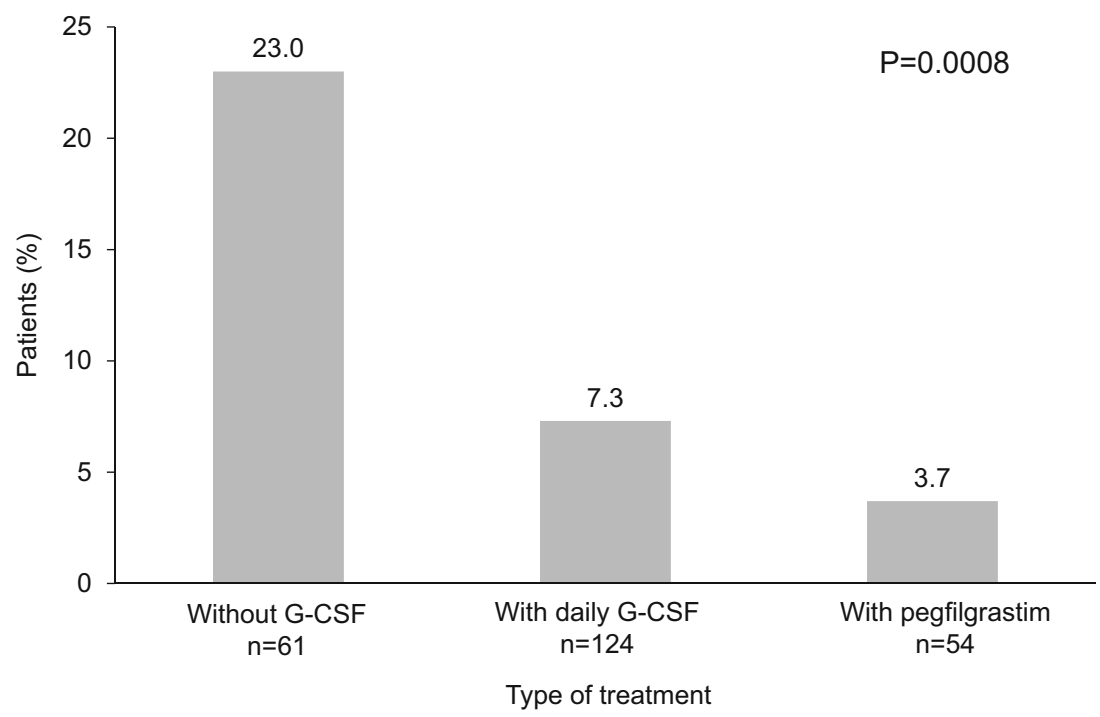

[13]. Moreover, the incidence of FN in cycle 1 and timing of FN onset in Japanese patients with B-NHL reported herein is similar to that reported in previous studies $[3,8,15,16]$.

Table 2 Incidence of FN in cycle 1 by patient characteristics

\begin{tabular}{|c|c|c|c|}
\hline Characteristic & $\begin{array}{l}N=239 \\
n / N\end{array}$ & $(\%)$ & $\begin{array}{l}\text { Chi-square } \\
(P \text { value })\end{array}$ \\
\hline \multicolumn{4}{|l|}{ Age } \\
\hline$<65$ years & $6 / 115$ & (5.2) & 6.5051 \\
\hline$\geq 65$ years & $19 / 124$ & (15.3) & $(0.0108)^{\mathrm{a}}$ \\
\hline \multicolumn{4}{|l|}{ Albumin } \\
\hline$<3.5 \mathrm{~g} / \mathrm{dL}$ & $14 / 53$ & (26.4) & 18.5092 \\
\hline$\geq 3.5 \mathrm{~g} / \mathrm{dL}$ & $11 / 186$ & $(5.9)$ & $(<0.0001)^{\mathrm{a}}$ \\
\hline \multicolumn{4}{|l|}{ Hemoglobin } \\
\hline$<12 \mathrm{~g} / \mathrm{dL}$ & $19 / 86$ & $(22.1)$ & 19.4095 \\
\hline$\geq 12 \mathrm{~g} / \mathrm{dL}$ & $6 / 153$ & $(3.9)$ & $(<0.0001)^{\mathrm{a}}$ \\
\hline \multicolumn{4}{|l|}{ Use of antibiotics } \\
\hline No & $1 / 156$ & $(<1)$ & 46.2425 \\
\hline Yes & $24 / 83$ & (28.9) & $(<0.0001)^{\mathrm{a}}$ \\
\hline \multicolumn{4}{|c|}{$\begin{array}{l}\text { With or without G-CSF treatment in cycle } 1 \text { (treatment before } \\
\text { development of FN) }\end{array}$} \\
\hline No & $14 / 61$ & $(23.0)$ & 13.6431 \\
\hline Yes & $11 / 178$ & $(6.2)$ & $(0.0002)^{\mathrm{a}}$ \\
\hline \multicolumn{4}{|c|}{ Use of G-CSF in cycle 1 (treatment before development of FN) } \\
\hline Without G-CSF & $14 / 61$ & $(23.0)$ & 14.1505 \\
\hline With daily G-CSF & $9 / 124$ & $(7.3)$ & $(0.0008)^{\mathrm{a}}$ \\
\hline With pegfilgrastim & $2 / 54$ & (3.7) & \\
\hline
\end{tabular}

Parameters shown in the table are those with significant changes only. The incidence of FN was 10.5\% (25 of 239) in cycle 1 and $13.0 \%$ (31 of $239)$ throughout all cycles. The majority of FN cases $(80.6 \% ; 25$ of 31$)$ occurred during cycle 1

$F N$, febrile neutropenia; $G$ - $C S F$, granulocyte colony-stimulating factors

${ }^{a}$ Data were analyzed using chi-squared test
In the present study, an increase in the prophylactic use of GCSF was observed, compared with the previous study [13]. This increase likely resulted from the implementation of the internal policy for pegfilgrastim use (Team G-LASTA), established based on the guidelines for use of G-CSF [4]. Both daily G-CSF and pegfilgrastim, administered for the prevention of FN, significantly reduced the incidence of FN compared with the group that did not receive G-CSF. Although no significant difference was observed between daily G-CSF and pegfilgrastim, the incidence of $\mathrm{FN}$ in patients treated with pegfilgrastim was numerically lower than that in patients treated with daily G-CSF. Additionally, we confirmed that the timing of daily G-CSF and pegfilgrastim administration (i.e., daily administration of G-CSF to patients with signs of FN and pegfilgrastim administration during the early phase of a chemotherapy cycle) in current clinical practice is comparable with what has been previously reported [14]. A possible reason why the incidence of FN was lower with pegfilgrastim than with daily-G-CSF is that daily G-CSF was administered later in the treatment cycle, when the signs of FN were confirmed, which may not be the optimal timing for G-CSF administration.

The presently identified risk factors for $\mathrm{FN}$ development in cycle 1 were similar to those reported previously $[8,9,13]$. No new risk factors for FN were identified, and thus, our results validate previous findings. This is valuable as it allows the identification of patients with a predisposition to develop FN and the implementation of an optimal prevention of FN with G-CSF in Japan.

Notably, patients with low ALC $\left(<1.0 \times 10^{9} / \mathrm{L}\right)$ showed a significantly higher incidence of FN than other patients. This finding suggests that patients with low ALC could be more prone to developing FN; thus, these patients should be carefully monitored. Several previous studies have indicated that low baseline blood cell counts, including pretreatment ALC, are predisposing factors for FN development [15, 17-20]. 
Fig. 2 Incidence of febrile neutropenia in cycle 1 by absolute lymphocyte count levels. $P=$ 0.0228 for absolute lymphocyte count $<1.0 \times 10^{9} / \mathrm{L}$ vs 1.0 to $2.0 \times 10^{9} / \mathrm{L}$ and $>2.0 \times 10^{9} / \mathrm{L}$ (chi-squared test)

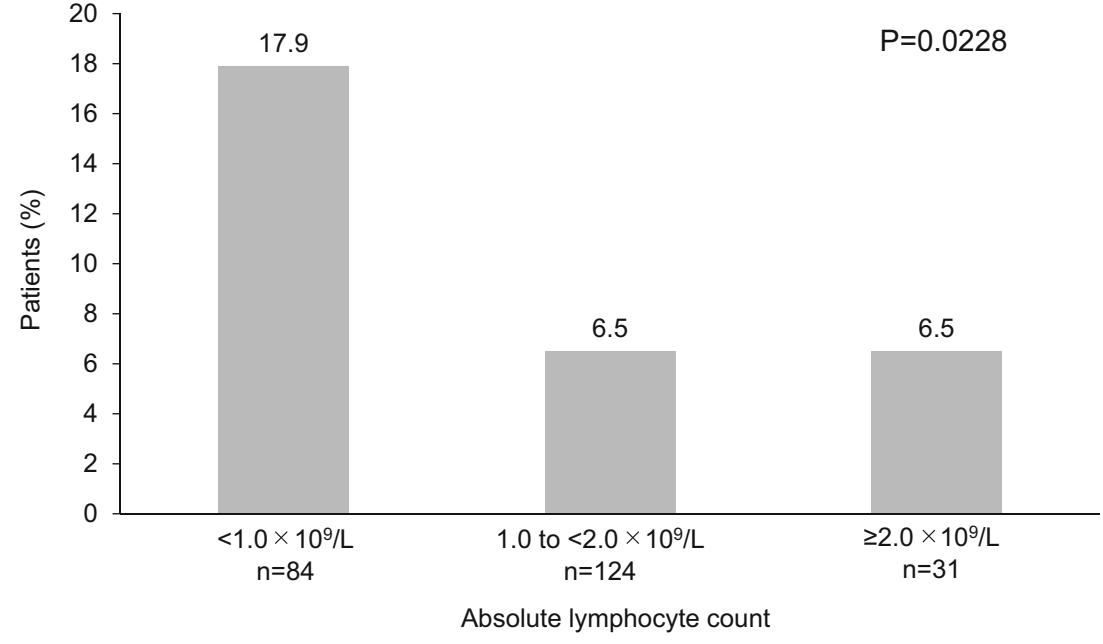

In a study by Jenkins et al., the authors developed a predictive model to identify patients at increased risk of FN following chemotherapy based on pretreatment hematological indices [17]. Patients in the highest risk group (ALC $\leq 1.5 \times 10^{9}$ / L) had a 3.4-fold increased risk of developing $\mathrm{FN}(P=0.001)$ and a 5.2-fold increased risk of cycle $1 \mathrm{FN}(P<0.001)$ [17]. In an earlier study by the same authors [18], the group of patients with the lowest values of ALC, which comprised $6 \%$ of the total population, had a risk of $\mathrm{FN}(21 \%)$ over fivefold greater than patients in the lowest risk group.

Table 3 Factors associated with the risk of febrile neutropenia in cycle 1 (univariate and multivariate analysis, $N=239$ )

\begin{tabular}{|c|c|c|c|c|c|c|}
\hline & \multicolumn{3}{|c|}{ Univariate analysis } & \multicolumn{3}{|c|}{ Multivariate analysis } \\
\hline & OR & $95 \% \mathrm{CI}$ & $P$ value & OR & $95 \% \mathrm{CI}$ & $P$ value \\
\hline Age ( $<65$ years, $\geq 65$ years $)$ & 3.287 & $1.263,8.551$ & 0.0147 & 4.265 & $1.242,14.639$ & 0.0212 \\
\hline Sex (male, female) & 1.366 & $0.587,3.177$ & 0.4688 & - & - & - \\
\hline ECOG PS $(0-1,2-4)$ & 2.239 & $0.448,11.183$ & 0.3259 & - & - & - \\
\hline BMI $\left(<23 \mathrm{~kg} / \mathrm{m}^{2}, \geq 23 \mathrm{~kg} / \mathrm{m}^{2}\right)$ & 0.487 & $0.195,1.215$ & 0.1229 & - & - & - \\
\hline Pathological diagnosis (DLBCL, follicular lymphoma) & 0.381 & $0.085,1.705$ & 0.2067 & - & - & - \\
\hline Pathological diagnosis (DLBCL, transformed DLBCL) & 1.349 & $0.278,6.557$ & 0.7103 & - & - & - \\
\hline Pathological diagnosis (DLBCL, other) & 0.645 & $0.141,2.957$ & 0.5728 & - & - & - \\
\hline Stage (I-II, III-IV) & 1.864 & $0.771,4.504$ & 0.1665 & - & - & - \\
\hline Bone marrow infiltration (no, yes) & 1.979 & $0.680,5.759$ & 0.2103 & - & - & - \\
\hline Diabetes mellitus (no, yes) & 0.404 & $0.052,3.148$ & 0.3871 & - & - & - \\
\hline Hepatic or renal diseases (no, yes) & 2.783 & $0.712,10.874$ & 0.1411 & - & - & - \\
\hline Albumin $(\geq 3.5 \mathrm{~g} / \mathrm{dL},<3.5 \mathrm{~g} / \mathrm{dL})$ & 5.711 & $2.410,13.531$ & $<0.0001$ & 5.081 & $1.517,17.015$ & 0.0084 \\
\hline Total bilirubin $(<1 \mathrm{mg} / \mathrm{dL}, \geq 1 \mathrm{mg} / \mathrm{dL})$ & 0.644 & $0.081,5.144$ & 0.6783 & & & \\
\hline Hemoglobin $(\geq 12 \mathrm{~g} / \mathrm{dL},<12 \mathrm{~g} / \mathrm{dL})$ & 6.948 & $2.654,18.186$ & $<0.0001$ & 7.973 & $2.339,27.179$ & 0.0009 \\
\hline Absolute neutrophil count $\left(\geq 2.69 \times 10^{9} / \mathrm{L},<2.69 \times 10^{9} / \mathrm{L}\right)$ & 0.758 & $0.247,2.322$ & 0.6272 & - & - & - \\
\hline Absolute lymphocyte count $\left(\geq 0.71 \times 10^{9} / \mathrm{L},<0.71 \times 10^{9} / \mathrm{L}\right)$ & 2.605 & $1.071,6.336$ & 0.0347 & - & - & - \\
\hline Relative dose intensity $(\geq 85 \%,<85 \%)$ & 1.528 & $0.652,3.581$ & 0.3290 & & & \\
\hline Prophylaxis with G-CSF (no, yes) & 0.271 & $0.062,1.188$ & 0.0834 & & & \\
\hline Prophylaxis with daily G-CSF (no, yes) & 0.263 & $0.106,0.649$ & 0.0037 & 0.059 & $0.015,0.230$ & $<0.0001$ \\
\hline Prophylaxis with pegfilgrastim (no, yes) & 0.129 & $0.028,0.598$ & 0.0089 & 0.017 & $0.002,0.119$ & $<0.0001$ \\
\hline
\end{tabular}

Although BMI and presence/absence of complications did not show statistical significance in univariate analysis, these were included in the multivariate analysis because they are considered clinically significant factors

$B M I$, body mass index; $C I$, confidence interval; $D L B C L$, diffuse large B-cell lymphoma; ECOG PS, Eastern Cooperative Oncology Group performance status; $F N$, febrile neutropenia; $G-C S F$, granulocyte colony-stimulating factors; $O R$, odds ratio 
Table 4 Candidate risk factors for febrile neutropenia in cycle 1 $(N=239)$

\begin{tabular}{lccccc}
\hline & $\begin{array}{l}\text { Parameter } \\
\text { estimate }\end{array}$ & $\begin{array}{l}\text { Standard } \\
\text { error }\end{array}$ & OR & 95\% CI & $P$ value \\
\hline Multivariate: stepwise, $P=0.20$ & & & & & \\
Age $(<65$ years, $\geq 65$ years) & 1.4078 & 0.6438 & 4.087 & $1.157,14.433$ & 0.0288 \\
Diabetes mellitus (no, yes) & -1.9398 & 1.3622 & 0.144 & $0.010,2.075$ & 0.1544 \\
Albumin $(\geq 3.5 \mathrm{~g} / \mathrm{dL},<3.5 \mathrm{~g} / \mathrm{dL})$ & 1.6794 & 0.6266 & 5.362 & $1.570,18.310$ & 0.0074 \\
Hemoglobin $(\geq 12 \mathrm{~g} / \mathrm{dL},<12 \mathrm{~g} / \mathrm{dL})$ & 2.1435 & 0.6364 & 8.529 & $2.450,29.692$ & 0.0008 \\
Prophylaxis with daily G-CSF (no, yes) & -2.885 & 0.7033 & 0.056 & $0.014,0.222$ & $<0.0001$ \\
Prophylaxis with pegfilgrastim (no, yes) & -4.1641 & 0.9914 & 0.016 & $0.002,0.109$ & $<0.0001$ \\
Estimated FN incidence, 30.4\% (95\% CI, 17.2-43.1\%) & & & & \\
\hline
\end{tabular}

$C I$, confidence interval; $F N$, febrile neutropenia; $G-C S F$, granulocyte colony-stimulating factors; $O R$, odds ratio
As a possible reason for such outcomes, Jenkins et al. proposed that low white blood cell counts, such as ALC, may reflect an insufficient bone marrow reserve. Relatively high bone marrow concentrations of the chemokine, stromal cellderived factor 1 (SDF-1), promote retention of B lymphocyte and neutrophils in the marrow [21]. Thus, together with limiting amounts of endogenous G-CSF, increased SDF-1 levels in bone marrow may be another potential reason for low pretreatment ALC levels [18]. Additionally, patients with lower baseline ALC will likely have low B lymphocyte count as well, which may be a cause of the increased incidence of FN [21]. Another study reported that the incidence of FN was significantly higher in patients with lymphocyte counts $\leq$ $700 / \mu \mathrm{L}$ at chemotherapy day $5(P=0.0001)$, and a day 5 lymphocyte count $\leq 700 / \mu \mathrm{L}$ was identified as an independent risk factor for FN by logistic regression analysis [22].

In the guidelines, R-CHOP therapy is categorized as an intermediate risk for $\mathrm{FN}$ (with an FN incidence between 10 and 20\%) [4]. The present study reported an incidence of $23.0 \%$, which is categorized as high risk of developing FN (an FN incidence of $20 \%$ or greater), in patients not receiving G-CSF. Thus, the appropriate use of G-CSF seems relevant for preventing FN among patients receiving $\mathrm{R}-\mathrm{CHOP}$.

In the present study, the simulation analysis was done for reference. The resulting FN incidence was $30.4 \%$, which is more than $10 \%$ higher compared with the result of the simulation analysis in the previous study (16.2\%) [13]. However, the reason for the difference of nearly $10 \%$ in the incidence of FN in patients not receiving prophylactic G-CSF between the previous and current studies remains unclear. In the simulation analysis in the present study, we used the risk factors that were not identified in the previous study. The estimated odds ratio for hemoglobin was high, and there was a relatively strong relationship between hemoglobin and the use of G-CSF. In the group receiving G-CSF, compared with the group not receiving G-CSF, the proportion of patients with hemoglobin $<12 \mathrm{~g} /$ $\mathrm{dL}$ and albumin $<3.5 \mathrm{~g} / \mathrm{dL}$, who were at high risk for developing FN, was higher. Lower levels of hemoglobin and albumin may indicate a compromised systemic condition, which can further exacerbate the condition and lead to a higher incidence of FN. This could potentially explain a higher incidence of FN in the group receiving G-CSF compared with the group not receiving G-CSF.

This study has several limitations, such as those inherent to observational and retrospective studies. This study was conducted at a single center which hampers the generalizability of the results. As cycle 2 and subsequent cycles were administered on an outpatient basis, it is possible that the detection of FN was not as accurate as in cycle 1 (inpatient treatment). Further, the results may have been influenced by the medical care preferences (e.g., all patients were hospitalized during cycle 1) which are specific to routine clinical practice in Japan. The sample size was small, which may have precluded some results from reaching statistical significance. Finally, HIV-positive patients were excluded from the study because in Japan, HIV-positive patients are treated at specifically designated hospitals, and the study site was not one of these centers.

\section{Conclusions}

In the present study, the incidence of FN in B-NHL patients who received R-CHOP regimen was $10.5 \%$ in cycle 1 of chemotherapy and $13.0 \%$ in all cycles. Additionally, age $\geq$ 65 years, albumin $<3.5 \mathrm{~g} / \mathrm{dL}$, hemoglobin $<12 \mathrm{~g} / \mathrm{dL}$, and lack of prophylaxis with daily G-CSF and pegfilgrastim were identified as significant risk factors of FN during cycle 1. Patients receiving daily G-CSF and pegfilgrastim had a significantly lower incidence of FN compared with those who did not receive G-CSF. The incidence of FN in patients receiving pegfilgrastim was lower than that in patients treated with daily G-CSF, although this difference was not statistically significant. A future large-scale randomized study should be performed to further validate the results of this small-scale retrospective study. 
Acknowledgments The authors would like to thank Keyra Martinez Dunn, MD, of Edanz Medical Writing for providing medical writing services, which was funded by Kyowa Hakko Kirin Co., Ltd., through EMC K.K. in accordance with Good Publication Practice (GPP3) guidelines (http://www.ismpp.org/gpp3).

Funding information Funding support for this research was provided by the Kyowa Hakko Kirin Co. Ltd.

\section{Compliance with ethical standards}

Conflict of interest M Yokoyama reports personal fees (for consultancy) from Chugai Pharmaceutical, outside the submitted work; N Nishimura reports personal fees (for consultancy) from Chugai Pharmaceutical, outside the submitted work; Y Mishima reports personal fees (for consultancy) from Chugai Pharmaceutical, outside the submitted work; T Nukada and T Nomura report personal fees (for employment) from Kyowa Hakko Kirin, during the conduct of the study; K Hatake reports personal fees (for expert testimony) from Eisai and Otsuka Pharmaceutical, grants from AbbVie, Gilead Sciences, Celgene, Solasia Pharma, Pfizer, BristolMyers Squibb, Janssen Pharmaceutical, Ono Pharmaceutical, and Chugai Pharmaceutical, personal fees (for speakers' bureau and others) from Takeda Pharmaceutical and Taiho Pharmaceutical, personal fees (for speakers' bureau) from Celgene, and personal fees (for others) from Kyowa Hakko Kirin, outside the submitted work. Y Kusano, A Nishihara, N Inoue and Y Terui have nothing to disclose.

Research involving human participants-ethical approval The study has been approved by the appropriate institutional research ethics committee and has been performed in accordance with the ethical standards as described in the 1964 Declaration of Helsinki and its later amendments or comparable ethical standards.

Informed consent For this type of study, formal consent is not required.

Open Access This article is distributed under the terms of the Creative Commons Attribution 4.0 International License (http:// creativecommons.org/licenses/by/4.0/), which permits unrestricted use, distribution, and reproduction in any medium, provided you give appropriate credit to the original author(s) and the source, provide a link to the Creative Commons license, and indicate if changes were made.

\section{References}

1. Crawford J, Dale DC, Lyman GH (2004) Chemotherapy-induced neutropenia: risks, consequences, and new directions for its management. Cancer 100:228-237

2. Lyman GH, Poniewierski MS, Culakova E (2016) Risk of chemotherapy-induced neutropenic complications when treating patients with non-Hodgkin lymphoma. Expert Opin Drug Saf 15: 483-492

3. Weycker D, Barron R, Kartashov A, Legg J, Lyman GH (2014) Incidence, treatment, and consequences of chemotherapy-induced febrile neutropenia in the inpatient and outpatient settings. J Oncol Pharm Pract 20:190-198

4. Japan Society of Clinical Oncology (2013) Clinical practice guidelines. http://www.jsco-cpg.jp/item/30/index.html. Accessed 6 May 2019
5. Aapro MS, Bohlius J, Cameron DA, Dal Lago L, Donnelly JP, Kearney N, Lyman GH, Pettengell R, Tjan-Heijnen VC, Walewski J, Weber DC, Zielinski C, European Organisation for Research and Treatment of Cancer (2011) 2010 update of EORTC guidelines for the use of granulocyte-colony stimulating factor to reduce the incidence of chemotherapy-induced febrile neutropenia in adult patients with lymphoproliferative disorders and solid tumours. Eur J Cancer 47:8-32. https://doi.org/10.1016/j. ejca.2010.10.013

6. Wang L, Baser O, Kutikova L, Page JH, Barron R (2015) The impact of primary prophylaxis with granulocyte colonystimulating factors on febrile neutropenia during chemotherapy: a systematic review and meta-analysis of randomized controlled trials. Support Care Cancer 23:3131-3140

7. Mhaskar R, Clark OA, Lyman G, Engel Ayer Botrel T, Morganti Paladini L, Djulbegovic B (2014) Cochrane Database Syst Rev 10: CD003039

8. Chao C, Rodriguez R, Page JH, Yang SJ, Huynh J, Chia VM (2015) History of chronic comorbidity and risk of chemotherapy-induced febrile neutropenia in patients with non-Hodgkin lymphoma not receiving granulocyte colony-stimulating factor prophylaxis. Leuk Lymphoma 56:72-79

9. Lyman GH, Morrison VA, Dale DC, Crawford J, Delgado DJ, Fridman M, OPPS Working Group; ANC Study Group (2003) Risk of febrile neutropenia among patients with intermediategrade non-Hodgkin's lymphoma receiving CHOP chemotherapy. Leuk Lymphoma 44:2069-2076

10. Lyman GH, Poniewierski MS (2017) A patient risk model of chemotherapy-induced febrile neutropenia: lessons learned from the ANC Study Group. J Natl Compr Cancer Netw 15:1543-1550

11. Aapro M, Boccia R, Leonard R, Camps C, Campone M, Choquet S, Danova M, Glaspy J, Hus I, Link H, Sliwa T, Tesch H, Valero V (2017) Refining the role of pegfilgrastim (a long-acting G-CSF) for prevention of chemotherapy-induced febrile neutropenia: consensus guidance recommendations. Support Care Cancer 25:32953304

12. Truong J, Lee EK, Trudeau ME, Chan KK (2016) Interpreting febrile neutropenia rates from randomized, controlled trials for consideration of primary prophylaxis in the real world: a systematic review and meta-analysis. Ann Oncol 27:608-618

13. Yokoyama M, Kusano Y, Takahashi A, Inoue N, Ueda K, Nishimura N, Mishima Y, Terui Y, Nukada T, Nomura T, Hatake $\mathrm{K}$ (2017) Incidence and risk factors of febrile neutropenia in patients with non-Hodgkin B-cell lymphoma receiving R-CHOP in a single center in Japan. Support Care Cancer 25:3313-3320

14. Crawford J, Becker PS, Armitage JO, Blayney DW, Chavez J, Curtin P, Dinner S, Fynan T, Gojo I, Griffiths EA, Hough S, Kloth DD, Kuter DJ, Lyman GH, Mably M, Mukherjee S, Patel S, Perez LE, Poust A, Rampal R, Roy V, Rugo HS, Saad AA, Schwartzberg LS, Shayani S, Talbott M, Vadhan-Raj S, Vasu S, Wadleigh M, Westervelt P, Burns JL, Pluchino L (2017) Myeloid growth factors, version 2.2017, NCCN clinical practice guidelines in oncology. J Natl Compr Cancer Netw 15:1520-1541

15. Lyhman GH, Kuderer NM, Crawford J, Wolff DA, Culakova E, Poniewierski MS, Dale DC (2011) Predicting individual risk of neutropenic complications in patients receiving cancer chemotherapy. Cancer 117:1917-1927

16. Lyman GH, Delgado DJ (2003) Risk and timing of hospitalization for febrile neutropenia in patients receiving CHOP, CHOP-R, or CNOP chemotherapy for intermediate-grade non-Hodgkin lymphoma. Cancer 98:2402-2409

17. Jenkins P, Scaife J, Freeman S (2012) Validation of a predictive model that identifies patients at high risk of developing febrile neutropaenia following chemotherapy for breast cancer. Ann Oncol 23:1766-1771 
18. Jenkins P, Freeman S (2009) Pretreatment haematological laboratory values predict for excessive myelosuppression in patients receiving adjuvant FEC chemotherapy for breast cancer. Ann Oncol 20: $34-40$

19. Schwenkglenks M, Pettengell R, Jackisch C, Paridaens R, Constenla M, Bosly A, Szucs TD, Leonard R (2011) Risk factors for chemotherapy-induced neutropenia occurrence in breast cancer patients: data from the INC-EU Prospective Observational European Neutropenia Study. Support Care Cancer 19:483-490

20. Lyman GH, Abella E, Pettengell R (2014) Risk factors for febrile neutropenia among patients with cancer receiving chemotherapy: a systematic review. Crit Rev Oncol Hematol 90:190-199
21. Cézé N, Thibault G, Goujon G, Viguier J, Watier H, Dorval E, Lecomte T (2011) Pre-treatment lymphopenia as a prognostic biomarker in colorectal cancer patients receiving chemotherapy. Cancer Chemother Pharmacol 68:1305-1313

22. Choi CW, Sung HJ, Park KH, Yoon SY, Kim SJ, Oh SC, Seo JH, Kim BS, Shin SW, Kim YH, Kim JS (2003) Early lymphopenia as a risk factor for chemotherapy-induced febrile neutropenia. Am J Hematol 73:263-266

Publisher's note Springer Nature remains neutral with regard to jurisdictional claims in published maps and institutional affiliations. 